\title{
Chondroblastoma of the Medial Malleolus: A Very Rare Case with Radiopathological Correlation
}

\author{
Sujay K Das ${ }^{1}$, Lokesh Meena ${ }^{1 *}$, Ravi S Singh ${ }^{1}$, Pankaj Sharma ${ }^{1}$, Pranoy Paul ${ }^{2}$ and Mohit Dhingra ${ }^{3}$ \\ ${ }^{1}$ Department of Radiodiagnosis, AIIMS Rishikesh, India \\ ${ }^{2}$ Department of Pathology, AIIMS Rishikesh, India \\ ${ }^{3}$ Department of Orthopedics, AIIMS Rishikesh, India \\ *Corresponding author: Lokesh Meena, Department of Radiodiagnosis, AIIMS Rishikesh, India
}

\section{ARTICLE INFO}

Received: 幽 July 27, 2021

Published: 幽 August 04, 2021

Citation: Sujay K Das, Lokesh Meena, Ravi S Singh, Pankaj Sharma, Pranoy Paul, Mohit Dhingra. Chondroblastoma of the Medial Malleolus: A Very Rare Case with Radiopathological Correlation. Biomed J Sci \& Tech Res 37(5)-2021. BJSTR. MS.ID.006057.

Abbreviations: MRI: Magnetic Resonance Imaging; T1WI: T1 Weighted Image; T2WI: T2 Weighted Image; DWI: Diffusion Weighted Image; ADC: Apparent Diffusion Coefficient; PDFS: Proton Density Fat Saturated; T1FS: T1 Fat Saturated.

\section{ABSTRACT}

Tumors of the medial malleolus are a rare entity, with chondrogenic tumors of the medial malleolus being extremely rare. Very few cases of chondroblastoma of the medial malleolus are reported in the literature at present. We report a case of 29 years old male with swelling over the left medial malleolus. Radiological features favored the diagnosis of chondroblastoma, which was further confirmed by histopathological examination. The appearance of chondroblastoma at a very uncommon site has been discussed with its clinical and radiographic features, differential diagnosis and management. This case appears to be one of the very few reported cases of chondroblastoma occurring at medial malleolus. The present case report will increase the awareness of this rare tumor occurring at this unusual site, thereby avoiding any chance of misdiagnosis.

\section{Learning Objectives}

a) To describe the radiologic spectrum of chondroblastoma in a very uncommon site like medial malleolus, its differentials, and complications.

b) To define the diagnosis of chondroblastoma in an appropriate clinical setting.

c) To analyze the radiographic and MRI findings of chondroblastoma, to improve the diagnosis of this kind of tumor.

Keywords: Chondroblastoma; Medial Malleolus; Rare Tumor

\section{Background}

Chondroblastoma is an uncommon osseous neoplasm. It accounts for less than $1 \%$ of all bone tumors. Tumors of the medial malleolus are rare, and even rarer is the chondrogenic tumors of the medial malleolus [1]. The most common anatomical site involved by this tumor is the proximal humerus, followed by the distal femur, proximal femur, proximal tibia, talus and innominate bone, in descending order. The typical radiological finding is an eccentric osteolytic lesion, frequently accompanied by a thin sclerotic rim $[2,3]$.
The male to female ratio is greater than $2: 1$, with peak incidence at age of 16 years. The proximal tibia is the most common location; but there is near even distribution of cases between the proximal tibia, proximal humerus, proximal femur, and distal femur [4-6]. Malignant transformation in chondroblastoma has been described in only a few cases [7]. Patients usually present with pain and swelling, particularly if a pathological fracture is present [8]. Chondroblastoma is usually treated by simple curettage with bone grafting $[4,9]$. 


\section{Case Presentation}

A 29-year-old male, presented to the OPD of AIIMS Rishikesh with complaints of swelling localized to the left medial malleolus, for 1.5 years. He had pain over the swelling for 5 months. The pain was gradual in onset, intermittent and aggravated on walking, and relieved on rest and analgesic medications. The patient was able to walk normally without a limp and had no history of fever, significant weight loss or any significant medical history. He was admitted for the evaluation and management of his complaints. On physical evaluation, tenderness was present on the left medial malleolus, with a firm $6 \times 5 \mathrm{~cm}$ localized swelling, and normal temperature of the overlying skin. The swelling was noncompressible and non- reducible. The range of movements at the left ankle joint was normal, and pain-free in all planes. There was no lymphadenopathy, and systemic examination was normal. The laboratory findings were within the normal range. The patient was referred to the Department of Radiodiagnosis and Imaging for evaluation of the extent of the disease.

\section{Imaging}

Digital X-Ray (Figure 1) revealed an eccentrically located, well defined, radiolucent, lobulated lesion, with a thin sclerotic border, exhibiting a geographical pattern of bone destruction, involving left medial malleolus. A minimal periosteal reaction was seen, with likely soft tissue extension.
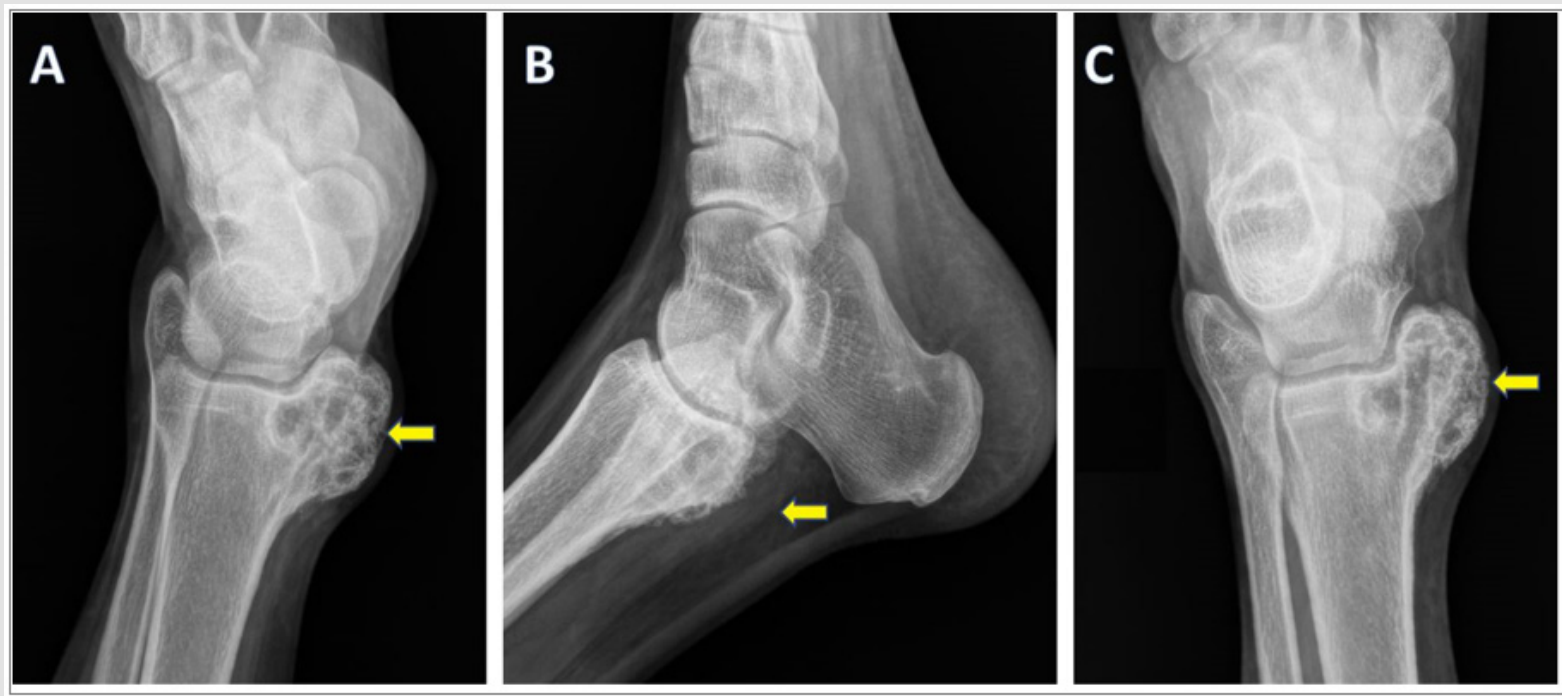

Figure 1: Radiography of left foot: internal oblique, lateral and AP views show an eccentrically located, radiolucent, lobulated lesion (marked with arrows), involving left medial malleolus. The lesion is well defined, lytic, with a thin sclerotic border, and exhibiting a geographical pattern of bone destruction. Cortical expansion and periosteal reaction are seen, with associated soft tissue extension.

MRI was performed using a 1.5 T MRI unit (Siemens Magnetom Aera). The MRI protocol included unenhanced multi-planar T1and T2-weighted and PDFS sequences, DWI and multiplanar postcontrast T1-weighted sequences. An intravenous contrast agent (Gadolinium) was administered. On unenhanced MR images, attenuation or intensity was classified as hypo, iso, or hyperintense, compared to the adjacent muscles. In post-gadolinium T1WI, the degree of enhancement of structures was classified as no enhancement, mild, moderate, or marked enhancement. Two experienced radiologists reviewed the MRI characteristics of the lesion. The following imaging features were documented: lesion size, location, shape, edge and intensity of the lesion at unenhanced and contrast-enhanced MRI, enhancement pattern, perineural spread, associated osseous abnormality, and presence of nodal or distant metastasis, depicted in Figures 2-5. Based on the clinical study and radiological data, chondroblastoma, giant cell tumour and chondromyxoid fibroma were kept as differentials.

\section{Histopathological Examination}

Core biopsy with Jamshidi needle was performed and representative photomicrographs are presented in Figure 6. 


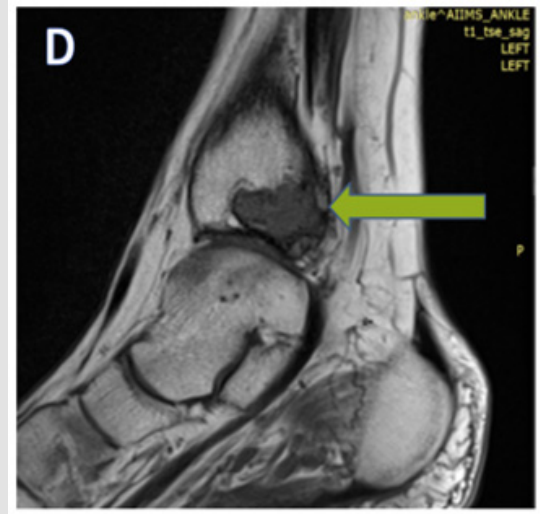

Sagittal T1WI

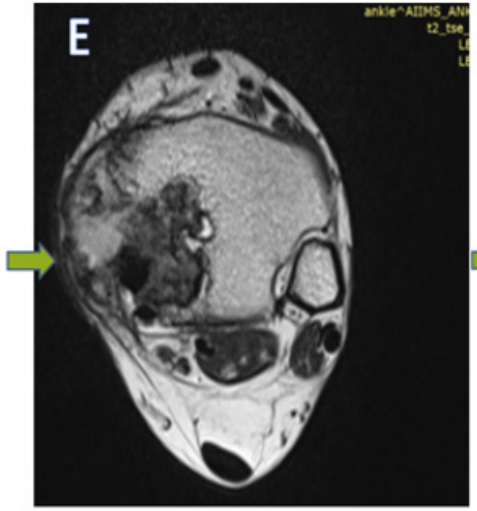

Axial T2WI

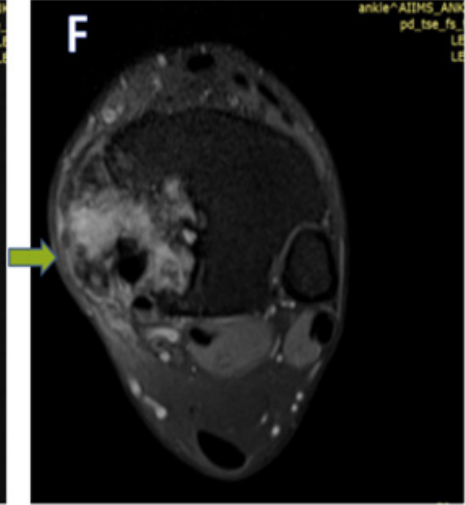

Axial PDFS image

Figure 2: An ill-defined lesion (marked with arrows) is seen involving the medial malleolus. It is showing a heterogeneously hypointense signal on T1WI, (D), and heterogeneously hyperintense signal on T2WI (E) and PDFS (F) images. A cortical breach is seen along the medial and inferior margin of the lesion, with extension into the adjacent soft tissue evident in images D\&E.
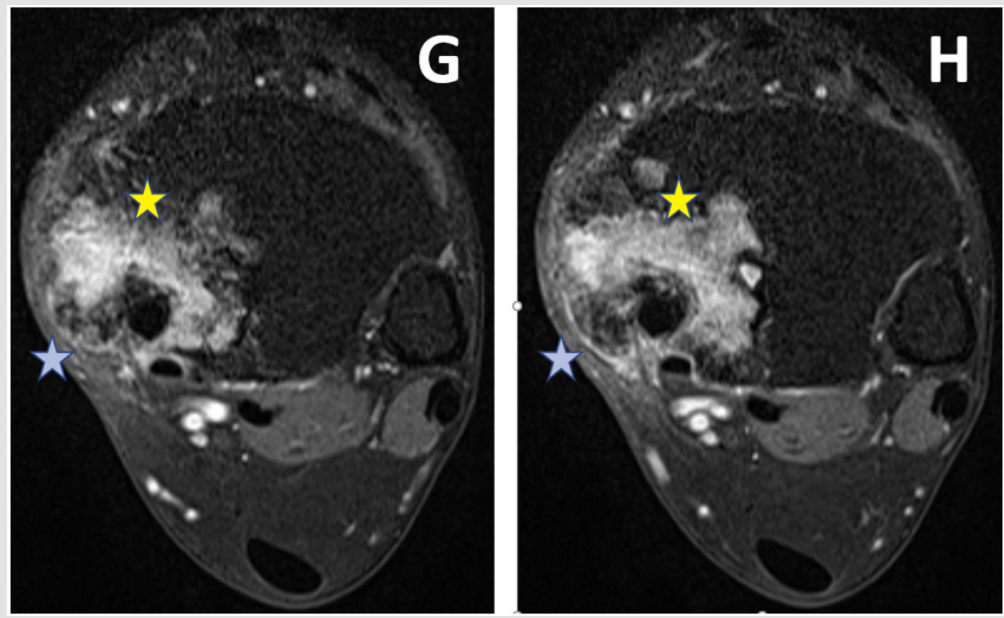

Figure 3: On post-gadolinium T1WI, heterogeneous enhancement is seen within the lesion (marked with Yellow stars. There is soft tissue extension (marked with blue stars). $\mathrm{G}$ is upper and $\mathrm{H}$ is a lower axial section.).
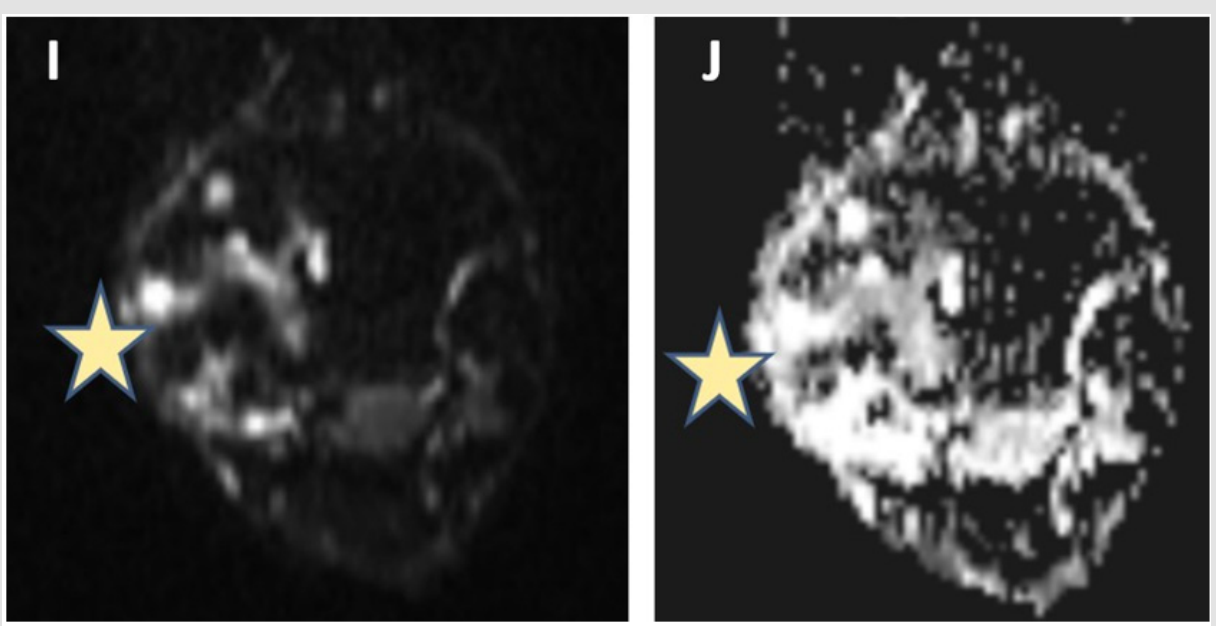

Figure 4: On diffusion-weighted imaging, peripheral areas of diffusion restriction (J) with low ADC (I) values are seen (marked with stars). 


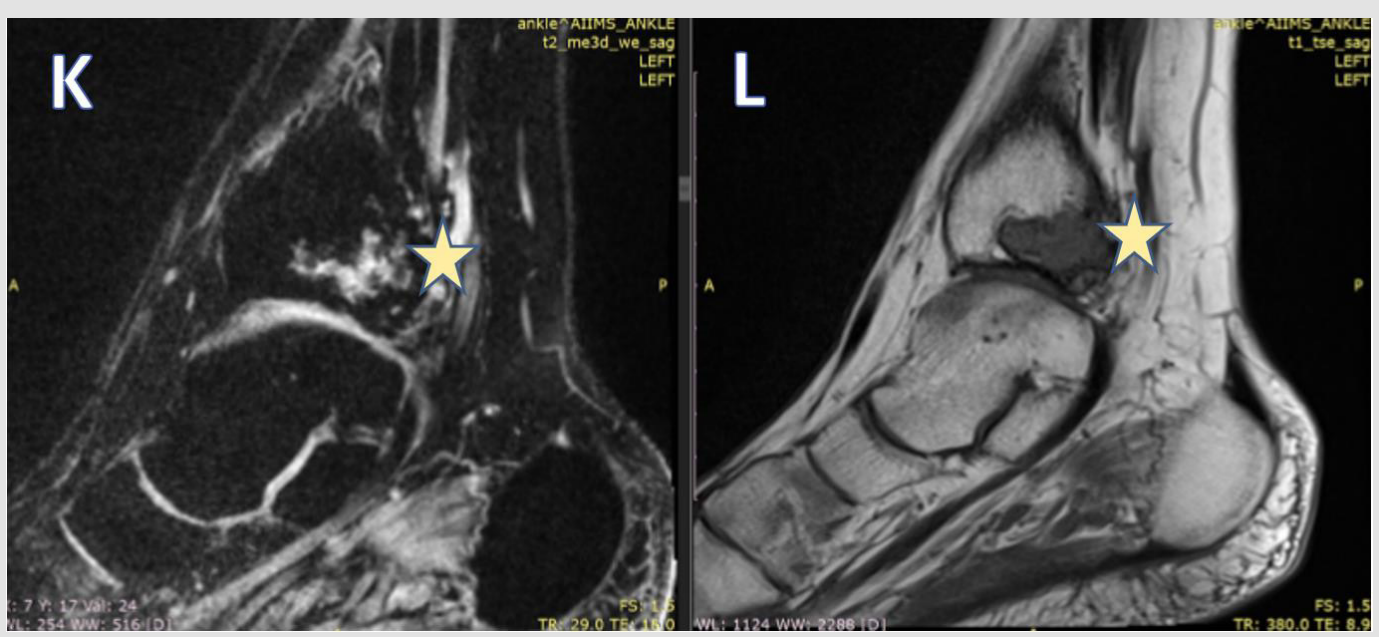

Figure 5: Sagittal T1FS (K) and T1W (L) images, showing: Posteriorly the tendons of the tibialis posterior and flexor digitorum are seen in close relation to the lesion (marked with arrows). The tendon of the tibialis posterior is completely engulfed. Posterior tibial vessels are well away from the lesion. The lesion is reaching up to the articular surface, however, no obvious extension is seen within the ankle joint. The ankle joint shows normal alignment. The joint space appears normal. The articular cartilage appears normal.
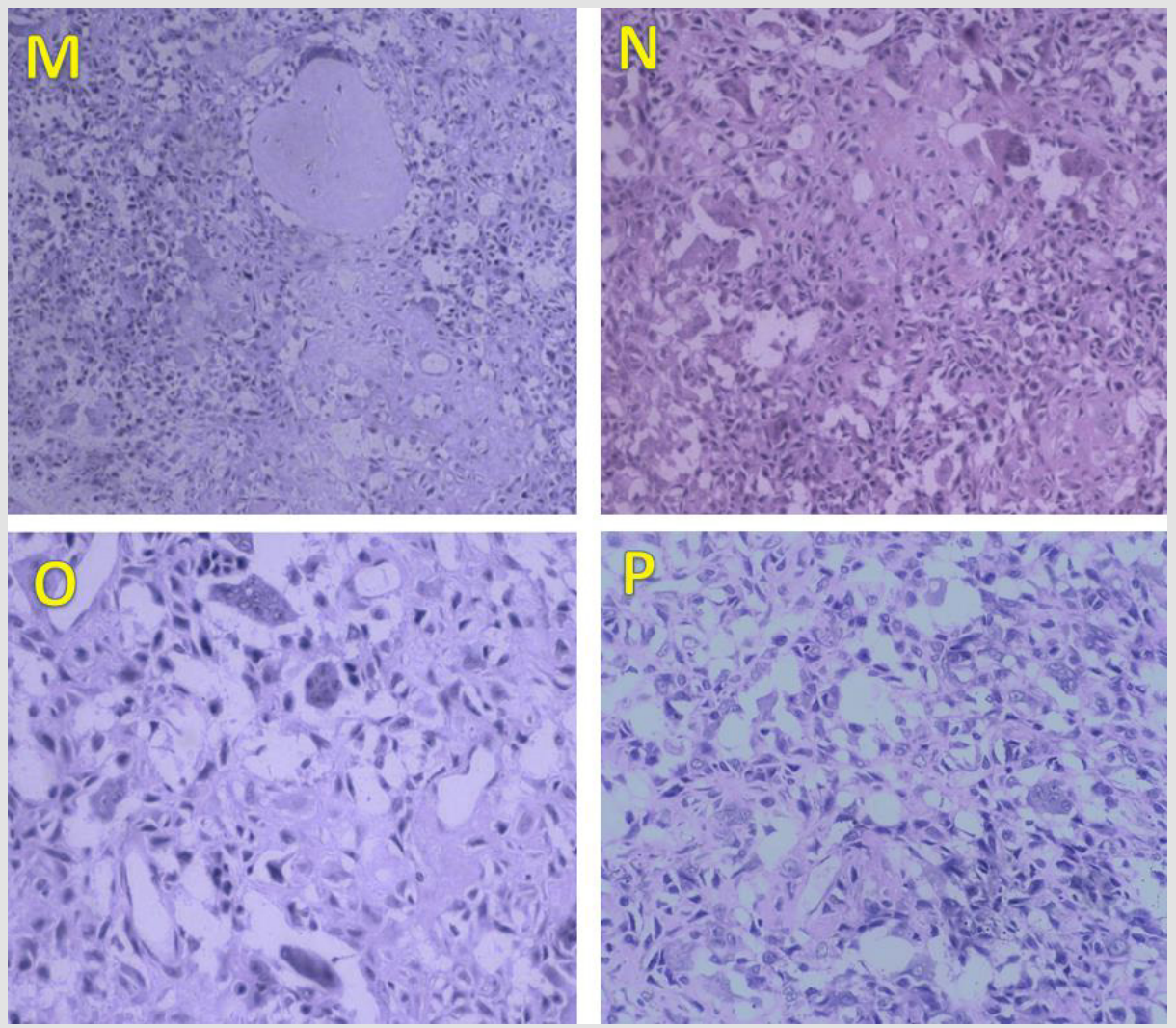

Figure 6: Photomicrographs of the lesion. Section examined from bony bits shows tumour tissue comprising of small to medium-sized spindle cells, along with bits of cortical bone $(\mathrm{H} \& \mathrm{E}, \times 100)$. The tumour cells have oval to spindle-shaped nuclei with grooving, bland nuclei, and abundant eosinophilic cytoplasm. Areas of osteoid and few chondroid areas are seen (M, $\mathrm{N})$. Numerous osteoclast-like giant cells $(\mathrm{O})$ are also seen scattered haphazardly with tumour cells. Chicken wire calcification is noted $(\mathrm{P})$ which is pathognomic of chondroblastoma. Mitosis is sparse. No necrosis was seen. These features are typical of chondroblastoma. 


\section{Management}

The patient was then taken up for surgery and extended curettage with contralateral iliac crest grafting was done. Curette material was sent for histopathological analysis, confirming the diagnosis of chondroblastoma. Follow up after 6 months showed no evidence of recurrence, with a normal range of movements at the ankle joint.

\section{Discussion}

Chondroblastoma is a rare tumor. It occurs in the second decade of life in up to $95 \%$ of cases, with a male: female ratio of 2:14. Chondroblastoma has a predilection for the epiphysis or apophysis of the distal femur, proximal humerus, proximal tibia. $10 \%$ of chondroblastoma affect the small bones of the hands and feet, with talus and calcaneum being the most common sites [5,6]. Pain and localized swelling, usually of several months' duration are the main and presenting complaints in patients with chondroblastoma $[2,7]$. On radiological examination, the lesion is usually eccentrically located, well defined, lytic, radiolucent, with a thin sclerotic border, exhibiting a geographical pattern of bone destruction [2-6]. Cortical expansion and periosteal reaction can be seen, which is uncommon in benign bone tumors [7,8]. Annual chest X-Rays must be done for 5 years after treatment, for detecting pulmonary metastasis, which is seen in 1 to $3 \%$ of patients [9].

Chondroblastoma exhibit a distinctive feature on MRI scans, which is a predominant low-to-intermediate signal intensity on T1-weighted images, with heterogeneous intermediate signal intensity on T2-weighted images, with only scattered foci of high signal intensity. Focal lobules of low, intermediate, and high signal intensity, most likely correspond to calcification, chondroid matrix, and fluid in the lesion. MRI can reveal an extension of tumor into the soft tissues which is usually rare and may also reveal an effusion of the joint [10].

On histopathology, chondroblastoma is seen to consist of immature chondrocytes, chondroblasts. It also contains a chondral or osteochondral matrix, which may calcify in a reticular pattern. Giant cells can also be found, but histological differentiation from giant cell tumors is possible due to the presence of a cartilaginous matrix and chondroblasts. In rare uncertain cases, immunohistochemical analysis showing cells positive for S-100 protein proves the chondral origin of these cells. Cartilaginous intercellular matrix with areas of focal calcification is typicalchicken wire calcification [11]. Biopsy of the lesion is mandatory to confirm the diagnosis.

Conditions mimicking chondroblastoma radiologically include bone abscess, giant cell tumor, chondromyxoid fibroma, osteoblastoma, enchondroma, intraosseous ganglion, clear cell chondrosarcoma, and osteonecrosis; while conditions mimicking chondroblastoma pathologically include giant cell tumor, enchondroma, clear cell chondrosarcoma and conventional chondrosarcoma [12]. More clearly demarcated margins of the lesion on radiography, and presence of chondroblasts and hyaline matrix on histopathological examination in this patient excluded osteoclastoma as a diagnosis. The epiphysis is a rare site for osteoblastoma and enchondroma, thereby excluding both. The younger age of this patient at the time of onset of symptoms excludes clear cell chondrosarcoma as a diagnosis, which commonly affects middle-aged patients. The young age of the patient at the time of onset of symptoms with a location in proximity to the articular surface rules out the diagnosis of an intraosseous ganglion. The absence of the classical radiological signs of osteonecrosis like the crescent sign and typical MRI findings in this patient excludes osteonecrosis. Absence of the myxoid component on histopathological examination rules out chondromyxoid fibroma.

The successful treatment comprises of aggressive curettage, phenolization, and bone cementation or bone autograft. A standard curettage procedure combined with cryosurgery is associated with the least recurrence rate. Various other surgical techniques, including open and endoscopic curettage, marginal resection, curettage with fat implantation, resection with radiofrequency ablation, and osteochondral autograft transfer are available nowadays [13-17]. Reconstruction of the bone defect as a result of surgery is another issue to address, and this can be taken care of with bone cement, and bone grafting. The most common method for reconstruction after curettage is a bone autograft, harvested from either iliac crest, as done in our case; or fibula with advantages of the improved rate of graft incorporation and lack of immunogenic concerns [14]. Advantages of bone cement for the reconstruction of bone defect includes immediate weight-bearing without risk of fracture, and an improved clinical and radiographic follow-up, which was observed in this patient also. Also, the bone graft is associated with longer surgical time, donor morbidity and may get resorbed, thereby making it difficult for differentiation of the graft from a tumor recurrence [18].

\section{Conclusion}

This case appears to be one of the very few reported cases of chondroblastoma occurring at the medial malleolus. This case report will increase the awareness of this rare tumor occurring at an unusual site thereby avoiding any chance of misdiagnosis. Aggressive curettage, phenolization and bone cement are preferred treatment methods. In this case, successful treatment consisted of an open biopsy followed by curettage and contralateral iliac crest grafting, without any recurrence at 6 months follow-up. 


\section{References}

1. Fletcher CDM, Unni KK, Mertens F (2013) World Health Organization classification of tumours pathology and genetics tumours of soft tissue and bone. Lyon: IARC Press.

2. Plum GE, Pugh DG (1958) Roentgenologic aspects of benign chondroblastoma of bone. Am J Roentgenol 79: 584-591.

3. Hudson TM, Hawkins IF (1981) Radiological evaluation of chondroblastoma. Radiology 139: 1-10.

4. Huvos AG, Marcove RC (1973) Chondroblastoma of bone. A critical review. Clin. Ortho 95: 300-302.

5. Schajowicz, Gallardo H (1970) Epiphysial chondroblastoma of bone. A clinicopathological study of sixty-nine cases. J Bone Joint Surg Br 52 205-226.

6. Dahlin DC, Ivins JC (1972) Benign chondroblastoma: a study of 125 cases. Cancer 30: 401-413.

7. Jesse A. Davila, Kimberly K. Amrami, Murali Sundaram, Mark C. Adkins, Krishnan K. Unni (2004) Chondroblastoma of the hands and feet. Skeletal Radiol 33: 582-587.

8. Kricun ME, Kricun R, Haskin ME (1977) Chondroblastoma of the calcaneus: radiographic features with emphasis on location. Am J Roentgenol 128: 613-616.

9. Kyriakos M, Land VJ, Penning HL, Parker SG (1985) Metastatic chondroblastoma. Report of a fatal case with a review of the literature on atypical, aggressive, and malignant chondroblastoma. Cancer 55: 1770-1789.

ISSN: 2574-1241

DOI: 10.26717/BJSTR.2021.37.006057

Pankaj Sharma. Biomed J Sci \& Tech Res

(c) (P) This work is licensed under Creative

Submission Link: https://biomedres.us/submit-manuscript.php
10. Jee, Park YK, McCauley TR, Choi KH, Ryu KN, et al. (1999) Chondroblastoma: MR characteristics with pathologic correlation. J Com Ass Tomo 23: 721-726.

11. Kaim AH, Hügli R, Bonél HM, Jundt G (2002) Chondroblastoma and clear cell chondrosarcoma: radiological and MRI characteristics with histopathological correlation. Skeletal Radiol 31(2): 88-95.

12. Akhtar K, Qadri S, Sen Ray P, Sherwani RK (2014) Cytological diagnosis of chondroblastoma: diagnostic challenge for the cytopathologist. BM] Case Reports.

13. Patrick P Lin, Arun Thenappan, Michael T Deavers, Valerae 0 Lewis, Alan W Yasko (2005) Treatment and prognosis of chondroblastoma. Clin Orthop Relat Res 438: 103-109.

14. Atalar H, Basarir K, Yildiz Y, Erekul S, Saglik Y (2007) Management of chondroblastoma: retrospective review of 28 patients. J Orthop Sci 12: 334-340.

15. Anderson AF, Ramsey JR (2003) Chondroblastoma of the talus treated with osteochondral autograft transfer from the lateral femoral condyle. Foot Ankle Int 24: 283-287.

16. Erickson JK, Rosenthal DI, Zaleske DJ, et al. (2001) Primary treatment of chondroblastoma with percutaneous radiofrequency heat ablation: report of three cases. Radiology 221: 463-468.

17. Sterling G, Wilson A (2002) Chondroblastoma of the talus: a case report J Foot Ankle Surg 41: 178-182.

18. Wade Sessions, Herrick J Siegel, James Thomas, Michael Pitt, Nasser SaidAl-Naief, et al. (2005) Chondroblastoma with associated aneurysmal bone cyst of the cuboid. J Foot Ankle Surg 44: 64-67.

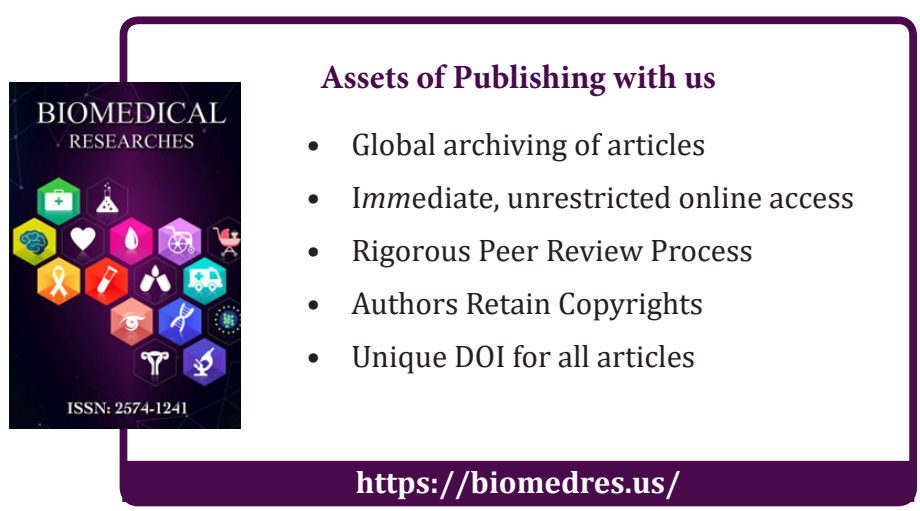

